\title{
Qualidade de melões (Cucumis melo L. var. cantalupensis Naud.), híbrido Torreon, produzidos em hidroponia e armazenados em embalagens de polietileno
}

\author{
Quality of Torreon hybrid melon fruits (Cucumis melo L. var. cantalupensis Naud.) grown in \\ hydroponic system and stored in polyethylene packaging
}

\author{
Auri Brackmann' ${ }^{1}$ Ana Cristina Eisermann² ${ }^{2}$ Ricardo Fabiano Hettwer Giehl ${ }^{3}$ \\ Evandro Binotto Fagan ${ }^{4}$ Sandro Luis Petter Medeiros ${ }^{2}$ \\ Cristiano André Steffens ${ }^{4}$
}

RESUMO

Este experimento teve por objetivos avaliar a permeabilidade de filmes de polietileno de diferentes espessuras e densidades ao $\mathrm{O}_{2}$ e ao $\mathrm{CO}_{2}$, a composição gasosa $\left(\mathrm{O}_{2}, \mathrm{CO}_{2}\right.$ e etileno) formada no interior das embalagens e a qualidade físico-química de melões (Cucumis melo L. var. cantalupensis Naud.), híbrido Torreon, produzidos no sistema hidropônico "Nutrient Film Technique" (NFT) e armazenados em embalagens de polietileno. Os tratamentos avaliados foram: (1) armazenamento refrigerado (sem uso de filme); (2) polietileno de baixa densidade (PEBD) de $40 \mu \mathrm{m}$; (3) $P E B D$ de $60 \mu \mathrm{m}$; (4) PEBD de $90 \mu \mathrm{m}$; (5) polietileno de média densidade (PEMD) de $40 \mu \mathrm{m}$; (6) PEMD de $60 \mu \mathrm{m}$. Os frutos permaneceram armazenados durante 25 dias a $3,8 \pm 0,2^{\circ} \mathrm{Ce}$ por mais dois dias a $20^{\circ} \mathrm{C}$. Os filmes de PEBD de 60 e $90 \mu \mathrm{m}$ e $o$ PEMD de $60 \mu \mathrm{m}$ apresentaram menor permeabilidade ao $\mathrm{CO}_{2}$, mantendo as maiores concentrações de $\mathrm{CO}_{2}$ nas embalagens. O filme de PEBD de $90 \mu \mathrm{m}$ apresentou menor permeabilidade ao $O_{2}$. A menor concentração de etileno foi obtida com o uso de PEBD de $40 \mu \mathrm{m}$. O uso de filmes reduziu drasticamente a perda de massa dos frutos, quando comparados aos frutos não embalados. Os frutos acondicionados na embalagem de PEBD de $40 \mu \mathrm{m}$ mantiveram uma maior firmeza da polpa após o período de armazenamento, não diferindo estatisticamente dos frutos armazenados em PEMD de $40 \mu \mathrm{m}$. Já a incidência de podridões foi significativamente menor nos frutos armazenados em PEMD de 60 $\mu \mathrm{m}$. De modo geral, os filmes avaliados mantêm semelhante a qualidade físicoquímica de melões híbrido Torreon produzidos hidroponicamente no sistema NFT.

Palavras-chave: atmosfera modificada, $\mathrm{O}_{2}, \mathrm{CO}_{2}$, etileno, permeabilidade.

\section{ABSTRACT}

This study was carried out to evaluate the permeability to $\mathrm{O}_{2}$ and $\mathrm{CO}_{2}$, and gas composition $\left(\mathrm{O}_{2}, \mathrm{CO}_{2}\right.$ and ethylene) inside packages of different thickness and density polyethylene films. Moreover, the physical and chemical quality of Torreon hybrid melon fruit (Cucumis melo L. var. cantalupensis Naud.) grown in a Nutrient Film Technique hydroponic system and stored within polyethylene packages were evaluated. Evaluated treatments were: (1) cold storage (control); packaging with (2) low-density polyethylene (LDPE)

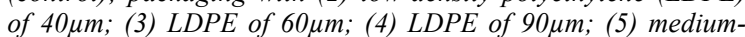
density polyethylene (MDPE) of $40 \mu \mathrm{m}$; (6) MDPE of $60 \mu \mathrm{m}$. Fruits were stored during 25 days at $3.8 \pm 0.2^{\circ} \mathrm{C}$ plus two days of shelf-life at $20^{\circ} \mathrm{C}$. LDPE of 60 and $90 \mu \mathrm{m}$ and MDPE of $60 \mu \mathrm{m}$ had lower $\mathrm{CO}_{2}$-permeability, allowing the higher $\mathrm{CO}_{2}$ concentrations inside the packages. LDPE of $90 \mu \mathrm{m}$ had the lowest $\mathrm{O}_{2}$-permeability. Lower ethylene concentration was observed in 40 $4 m-L D P E$ bags. Packaging decreased drastically fruit weight loss, when compared with non-packaged fruits. Flesh firmness was higher in fruits stored in $40 \mu m-L D P E$, but was not different from $40 \mu \mathrm{m}-M D P E$-stored fruits. Rot incidence in turn was significantly lower in fruits stored in $60 \mu m-L D P E$ In general, packaging of 'Torreon' melon fruit, grown in a hydroponic system, with the evaluated polyethylene films allowed similar physical and chemical quality.

Key words: modified atmosphere, $\mathrm{O}_{2}, \mathrm{CO}_{2}$, ethylene, permeability.

\section{INTRODUÇÃO}

No Brasil, o melão é amplamente produzido no Nordeste brasileiro, sendo a variedade inodorus a

\footnotetext{
${ }^{1}$ Departamento de Fitotecnia, Universidade Federal de Santa Maria (UFSM), 97105-900, Santa Maria, RS, Brasil. E-mail: brackman@ccr.ufsm.br. Autor para correspondência.

${ }^{2}$ Curso de Agonomia, CCR, UFSM, Santa Maria, RS, Brasil.

${ }^{3}$ Programa de Pós-graduação em Agronomia, UFSM, Santa Maria, RS, Brasil.

${ }^{4}$ Programa de Pós-graduação em Fitotecnia, Escola Superior de Agronomia "Luis de Queiroz" (ESALQ), Universidade de São Paulo (USP), Piracicaba, SP, Brasil.
} 
mais cultivada. Apesar de os frutos dessa cultivar apresentarem maior conservação pós-colheita e maior resistência ao transporte, são deficientes em termos de qualidade organoléptica. Nos últimos anos, a produção de melões da variedade cantalupensis tem aumentado significativamente no país. Esses melões apresentam sabor e aroma pronunciados, sendo bem aceitos no mercado interno e externo.

O cultivo de melões em sistema hidropônico proporciona maior produtividade, especialmente por permitir um controle mais rigoroso do aporte de água e de nutriente às plantas (FAGAN, 2005). Além disso, esse sistema permite a colheita de melões com melhor qualidade visual e sanitária. Melões produzidos em sistema hidropônico geralmente são colhidos quando se desprendem totalmente da planta, pois, segundo WELLES \& BUITELAAR (1988), o conteúdo de açucares é diretamente relacionado ao tempo em que os melões permanecem ligados à planta, porém o tempo de conservação pós-colheita é inversamente proporcional ao estádio de maturação.

Os melões são classificados como frutos climatéricos, sendo que os frutos da variedade cantalupensis apresentam um aumento drástico na síntese de etileno durante o climatério respiratório (SEYMOUR \& McGLASSON, 1993). Esses frutos, portanto, amadurecem rapidamente e possuem uma vida de prateleira curta, o que limita seu transporte e o seu potencial de expansão comercial. Como forma de prolongar a vida pós-colheita, pode-se fazer uso de baixas temperaturas, que permitem uma redução na taxa respiratória dos frutos. HARDENBURG et al. (1986) citam que a condição ótima de temperatura para o armazenamento de melões reticulados está na faixa de $2^{\circ} \mathrm{C}$ a $5^{\circ} \mathrm{C}$, com $95 \%$ de umidade relativa do ar. Porém, mesmo nessas condições, o período de armazenamento pode ficar limitado a 14 dias (SEYMOUR \& McGLASSON, 1993).

Outra técnica que pode ser utilizada para incrementar o efeito do frio é a atmosfera modificada (AM). A AM é conseguida com o uso de filmes de polietileno que retêm o $\mathrm{CO}_{2}$ liberado pela respiração dos frutos, aumentando a concentração deste gás de forma gradativa até determinado nível, onde começa a apresentar efeito inibitório sobre a respiração dos frutos (BRACKMANN et al.,1999). O polietileno é um polímero de composição química relativamente simples e com baixo custo, sendo muito utilizado na embalagem de alimentos em geral. É classificado como polietileno de baixa densidade (PEBD), quando a densidade varia de 0,910 a $0,940 \mathrm{~g} \mathrm{~cm}^{-3}$, e como polietileno de média densidade (PEMD), quando varia de 0,925 a $0,940 \mathrm{~g} \mathrm{~cm}^{-3}$ (SARANTÓPOULOS et al., 2002). O polietileno de baixa densidade possui alta permeabilidade ao vapor de água, quando comparado aos demais filmes plásticos, e geralmente constitui-se em barreira muito intensa ao $\mathrm{O}_{2}$ e ao $\mathrm{CO}_{2}$ na embalagem de frutas e hortaliças que possuem altas taxas respiratórias. O PEMD é um pouco mais resistente, mais rígido e menos permeável que o PEBD (SARANTÓPOULOS et al., 2002). Segundo KADER \& KE (1994), melões da variedade cantalupensis podem ser armazenados sob $2,0 \mathrm{kPa}$ de $\mathrm{O}_{2}$ e $15,0 \mathrm{kPa}$ de $\mathrm{CO}_{2}$.

Um fator muito importante que pode comprometer a qualidade dos frutos é a perda de massa, a qual pode ser atribuída à perda de umidade pela transpiração e de material de reserva, sendo um dos principais fatores limitantes da vida pós-colheita de melões. Esta última, cabe ressaltar, varia conforme a cultivar, os tratamentos pós-colheita e as condições e duração do armazenamento, entre outros (MAYBERRY \& HARTZ, 1992).

Os objetivos deste trabalho foram avaliar: (1) a permeabilidade de filmes de polietileno de diferentes espessuras e densidades ao $\mathrm{O}_{2}$ e ao $\mathrm{CO}_{2}$; (2) a composição gasosa $\left(\mathrm{O}_{2}, \mathrm{CO}_{2}\right.$ e etileno) formada nas embalagens; e (3) a qualidade físico-química de melões (Cucumis melo L. var. cantalupensis Naud.), híbrido Torreon, produzidos no sistema hidropônico "Nutrient Film Technique" (NFT) e armazenados em embalagens de polietileno.

\section{MATERIAL E MÉTODOS}

O experimento foi conduzido pelo Núcleo de Pesquisa em Pós-Colheita (NPP), juntamente com o Núcleo de Pesquisa em Ecofisiologia e Hidroponia (NUPECH), do Departamento de Fitotecnia da Universidade Federal de Santa Maria. Os frutos utilizados foram melões (Cucumis melo L. var. cantalupensis Naud.) híbrido Torreon, produzidos em sistema hidropônico "Nutrient Film Technique" (NFT), no período de janeiro a abril de 2004, conforme descrito em FAGAN (2005). Os frutos foram colhidos quando já haviam formado a zona de abscisão e soltado totalmente da planta. $\mathrm{O}$ delineamento experimental utilizado foi o delineamento inteiramente casualizado, com seis tratamentos de quatro repetições e unidade experimental composta por cinco frutos com massa de $970 \pm 130 \mathrm{~g}$ cada. No início do armazenamento, os frutos apresentavam firmeza da polpa de $17,4 \pm 2,4 \mathrm{~N}$, acidez titulável de $0,106 \pm 0,003 \mathrm{~g}$ ácido cítrico $100 \mathrm{~mL}^{-1}$, teor de 
sólidos solúveis totais de $10,4 \pm 0,6$ graus Brix, síntese de etileno de $44,20 \pm 4,60 \mu \mathrm{L} \mathrm{kg}^{-1} \mathrm{~h}^{-1}$ e respiração de $57,14 \pm 2,56 \mathrm{~mL} \mathrm{CO}_{2} \mathrm{~kg}^{-1} \mathrm{~h}^{-1}$.

No laboratório, os frutos passaram por um processo de seleção, sendo as amostras experimentais homogeneizadas. Em seguida, foram acondicionados em filmes de polietileno de diferentes espessuras e armazenados na temperatura de $3,8 \pm 0,2^{\circ} \mathrm{C}$ e $87 \pm 3,0 \%$ de umidade relativa do ar (UR). Os tratamentos avaliados foram: (1) armazenamento refrigerado sem o uso de filme (controle); (2) polietileno de baixa densidade (PEBD) de 40 $\mu \mathrm{m}$; (3) PEBD de $60 \mu \mathrm{m}$; (4) PEBD de $90 \mu \mathrm{m}$; (5) polietileno de média densidade (PEMD) de 40 $\mu \mathrm{m}$; (6) PEMD de $60 \mu \mathrm{m}$. Os frutos não foram resfriados e apresentavam, no início do armazenamento, temperatura da polpa de $20 \pm 1^{\circ} \mathrm{C}$. O objetivo desse procedimento foi obter mais rapidamente concentrações de $\mathrm{O}_{2}$ e de $\mathrm{CO}_{2}$, no interior das embalagens, próximas aos níveis sugeridos por KADER \& KE (1994).

Os frutos permaneceram durante 25 dias em ambiente refrigerado a $3,8 \pm 0,2^{\circ} \mathrm{C}$ e dois dias a $20 \pm 1^{\circ} \mathrm{C}$. Durante o período de armazenamento, foram determinadas as concentrações de $\mathrm{CO}_{2}, \mathrm{O}_{2}$ e etileno presentes no interior das embalagens, com intervalos de cinco dias entre uma leitura e outra. A concentração de etileno foi obtida pela injeção de amostras de $1 \mathrm{~mL}$ de cada embalagem em um cromatógrafo a gás marca Varian ${ }^{\circledR}$, modelo $3400 C X$, equipado com detector de ionização de chama (FID) e coluna Porapak N 80/100. Já as concentrações de $\mathrm{O}_{2}$ e $\mathrm{CO}_{2}$ foram determinadas por meio da circulação da composição gasosa de cada embalagem em um analisador de gases, com fluxo contínuo, da marca Agridatalog®.

Ao final do período de armazenamento, realizou-se a análise laboratorial, na qual foram avaliados os seguintes parâmetros: firmeza de polpa, determinada com penetrômetro manual e ponteira de $11 \mathrm{~mm}$ de diâmetro. Os frutos foram seccionados transversalmente e a firmeza obtida em diversos pontos da polpa dos frutos, no sentido longitudinal, da superfície seccionada. Sólidos solúveis totais (SST) foram obtidos com refratômetro manual, com compensação da temperatura para $20^{\circ} \mathrm{C}$. Os teores de SST foram expressos em graus Brix e a acidez total titulável (ATT) com a titulação de $10 \mathrm{ml}$ de suco diluído em $100 \mathrm{ml}$ de água e titulado com solução de $\mathrm{NaOH}$ até $\mathrm{pH} 8,1$. A perda de massa foi determinada pela pesagem dos frutos e a porcentagem de frutos podres por meio de uma avaliação visual, método este também utilizado para avaliar a presença de degenerescência na polpa.
A permeabilidade dos filmes foi determinada com o uso de câmaras de permeação com $4.500 \mathrm{~mL}$ de volume e uma área de permeação de $0,04524 \mathrm{~m}^{2}$, nas quais fixaram-se os filmes poliméricos, de forma a não ocorrer vazamentos entre o filme polimérico e a parede da câmara cilíndrica. Após a injeção de $\mathrm{N}_{2}$ e $\mathrm{CO}_{2}$, iniciouse a determinação dos gases, finalizando-a após cerca de 48 horas a $3,8 \pm 0,2^{\circ} \mathrm{C}$ e com UR do ar externa às câmaras de permeação de $87 \pm 3 \%$ e interna às câmaras de $<10 \%$, para determinar a concentração final. A permeabilidade dos filmes foi calculada pela fórmula $\mathrm{P}=((\mathrm{Ci} \times \mathrm{Vc} / 100)-(\mathrm{Cf} \times \mathrm{Vc} / 100)) /(\mathrm{Ap} \times \mathrm{t})$, sendo $\mathrm{P}$ a permeabilidade do filme polimérico dado em $\mathrm{mL}$ de gás $\mathrm{m}^{-2} \mathrm{dia}^{-1}$; Ci e Cf, a concentração inicial e final do gás, respectivamente, ambas dadas em \%; Vc, o volume interno da câmara de permeação, dado em mL; Ap, a área de permeação, dada em $\mathrm{m}^{2}$; e t, o tempo, dado em dias.

Os dados expressos em porcentagem foram transformados pela fórmula arc.senvx/100, para se efetuar a análise de variância. As médias foram comparadas entre si pelo teste Duncan em nível de 5\% de probabilidade de erro.

\section{RESULTADOS E DISCUSSÃO}

A permeabilidade dos filmes ao $\mathrm{O}_{2}$ e ao $\mathrm{CO}_{2}$ foi significativamente diferente (Tabela 1). Os filmes de PEBD de 60 e $90 \mu \mathrm{m}$ e o PEMD de $60 \mu \mathrm{m}$ apresentaram menor permeabilidade ao $\mathrm{CO}_{2}$, enquanto o filme de PEBD de $90 \mu \mathrm{m}$ foi menos permeável ao $\mathrm{O}_{2}$ (Tabela 1 ). De modo geral, filmes de PEMD têm sido considerados menos permeáveis que os de PEBD (SARANTÓPOULOS et al., 2002). No entanto, os resultados obtidos no presente trabalho demonstram uma maior influência da espessura e da densidade do filme sobre a permeabilidade ao $\mathrm{O}_{2}$, sendo a permeabilidade ao $\mathrm{CO}_{2}$ aparentemente mais afetada pela espessura do filme plástico que pela sua densidade (Tabela 1). Além disso, deve-se ressaltar que a permeabilidade dos filmes plásticos pode ser afetada pelos aditivos e plastificantes incorporados na matriz do filme, durante a sua fabricação.

As concentrações de $\mathrm{CO}_{2}$ e $\mathrm{O}_{2}$ no interior das embalagens variaram durante o período de armazenamento. As maiores concentrações de $\mathrm{CO}_{2} \mathrm{e}$ as menores de $\mathrm{O}_{2}$ foram observadas no 5o dia de armazenamento (Figuras 1A e 1B). No restante do período, as concentrações de $\mathrm{O}_{2}$ e $\mathrm{CO}_{2}$ aumentaram e diminuíram, respectivamente, independentemente do filme utilizado (Figuras 1A e 1B). Esse comportamento deve ter ocorrido em função de os frutos apresentarem 
Tabela 1 - Permeabilidade de filmes de polietileno ao dióxido de carbono e oxigênio. Santa Maria, RS. 2004.

\begin{tabular}{cccc}
\hline \multicolumn{2}{c}{ Filme de polietileno } & & \\
\hline Espessura $(\mu \mathrm{m})$ & Densidade & Permeabilidade ao $\mathrm{CO}_{2}\left(\mathrm{~mL} \mathrm{CO}_{2} \mathrm{~m}^{-2} \mathrm{dia}^{-1}\right)$ & Permeabilidade ao $\mathrm{O}_{2}\left(\mathrm{~mL} \mathrm{O}_{2} \mathrm{~m}^{-2} \mathrm{dia}^{-1}\right)$ \\
\hline 40 & Baixa & $633,8 \mathrm{a} *$ & $606,5 \mathrm{a}$ \\
60 & Baixa & $386,2 \mathrm{~b}$ & $304,6 \mathrm{~d}$ \\
90 & Baixa & $376,3 \mathrm{~b}$ & $260,8 \mathrm{e}$ \\
40 & Média & $451,5 \mathrm{a}$ & $560,8 \mathrm{~b}$ \\
60 & Média & $392,3 \mathrm{~b}$ & $438,0 \mathrm{c}$ \\
Média geral & & 448,1 & 434,1 \\
Coeficiente de variação (\%) & & 3,41 & 4,83 \\
\hline
\end{tabular}

*Médias não seguidas pela mesma letra, na vertical, diferem entre si pelo teste Duncan em nível de $5 \%$ de probabilidade de erro.

elevada respiração $\left(57,14 \pm 2,56 \mathrm{~mL} \mathrm{CO}_{2} \mathrm{~kg}^{-1} \mathrm{~h}^{-1}\right)$ e produção de etileno $\left(44,20 \pm 4,60 \mu \mathrm{L} \mathrm{kg}^{-1} \mathrm{~h}^{-1}\right)$ no início do armazenamento, pois foram embalados com temperatura de polpa de $20 \pm 1^{\circ} \mathrm{C}$. Além disso, durante o armazenamento, provavelmente o acúmulo de $\mathrm{CO}_{2}$ foi diminuindo, devido a sua menor produção, e as concentrações de $\mathrm{O}_{2}$ aumentando, em função do seu menor consumo, sendo ambas afetadas pela permeabilidade dos filmes.

Os filmes mais espessos, PEBD $60 \mu \mathrm{m}$ e PEBD 90 $\mu \mathrm{m}$, devido à menor permeabilidade (Tabela 1), mantiveram as maiores concentrações de $\mathrm{CO}_{2}$ nas embalagens, apresentando em média 14,5 e $14,1 \%$ de $\mathrm{CO}_{2}$, respectivamente, durante o período de armazenamento (Figura 1A). Nos primeiros 10 dias de armazenamento, as concentrações de $\mathrm{CO}_{2}$ no interior dessas embalagens estiveram acima de $15 \%$. Para KADER \& KE (1994), melões toleram concentrações de $\mathrm{CO}_{2}$ de até $15 \%$, sendo esse nível considerado adequado para o armazenamento de melões minimamente processados (PORTELA \& CANTWELL, 1998).

O filme de PEBD de $40 \mu \mathrm{m}$ apresentou a maior concentração de $\mathrm{O}_{2}$ durante todo o período de armazenamento, sendo que os demais filmes não apresentaram diferenças pronunciadas nas concentrações desse gás, a qual oscilou entre 4 e $9 \%$ (Figura 1B). A concentração de $\mathrm{O}_{2}$ no $5^{\circ}$ dia de armazenamento esteve próxima de $1 \%$ em todas as embalagens, sendo que KADER \& KE (1994) consideram que melões cantalupensis toleram concentrações de $\mathrm{O}_{2}$ de até $2 \%$. Apesar disso, os frutos não apresentaram "off-flavour" ou sabor alcoólico (dados não apresentados), provavelmente devido ao posterior aumento nas concentrações de $\mathrm{O}_{2}$, que alcançaram valores superiores a $4 \%$ a partir do $10^{\circ}$ dia de armazenamento (Figura 1B). Por isso, a restrição de $\mathrm{O}_{2}$ não teve grande efeito a partir dos 10 dias, pois segundo BEAUDRY (1999), níveis de $\mathrm{O}_{2}$ superiores a $5 \mathrm{kPa}$ não diminuem significativamente a atividade metabólica de tecidos vegetais, sendo gerada energia ainda suficiente para manter grande parte dos processos metabólicos primários e secundários, que ocorrem nas células.

No $10^{\circ}$ dia de armazenamento, observaramse as maiores concentrações de etileno nas embalagens de PEMD, sendo que, após esse período, decresceram acentuadamente (Figura 1C). Nas demais embalagens, não ocorreu esse comportamento. A menor concentração de etileno foi obtida com o uso de PEBD de $40 \mu \mathrm{m}$, provavelmente em função da maior permeabilidade desse filme a esse composto (parâmetro não avaliado).

$\mathrm{O}$ uso de filmes reduziu drasticamente a perda de massa dos frutos, quando comparados aos frutos não embalados (Tabela 2). A perda de umidade pode ser uma das principais causas da deterioração, pois acarreta não apenas perda quantitativa, mas provoca perdas qualitativas, devido ao murchamento do fruto (MAYBERRY \& HARTZ, 1992; MENEZES, 1996). A baixa permeabilidade oferecida pelo uso de filmes criou, dentro das embalagens, um ambiente com umidade relativa superior àquele externo, evitando a perda de água por transpiração.

Os frutos acondicionados na embalagem de PEBD de $40 \mu \mathrm{m}$ mantiveram uma maior firmeza da polpa 
Tabela 2 - Qualidade físico-química de melões, híbrido Torreon, produzidos em sistema hidropônico (NFT), após 25 dias de armazenamento a $3,8 \pm 0,2^{\circ} \mathrm{C}$ e mais dois dias a $20 \pm 1^{\circ} \mathrm{C}$. Santa Maria, RS. 2004.

\begin{tabular}{|c|c|c|c|c|c|c|}
\hline Tratamentos $^{1}$ & $\begin{array}{l}\text { Perda de } \\
\text { massa(\%) }\end{array}$ & $\begin{array}{l}\text { Firmeza da polpa } \\
\text { (N) }\end{array}$ & $\begin{array}{c}\text { Acidez tiulável } \\
\left.\text { (g ac. cítrico } 100 \mathrm{~mL}^{-1}\right)\end{array}$ & $\begin{array}{c}\text { SST } \\
\text { (graus Brix) }\end{array}$ & $\begin{array}{c}\text { Degenerescência } \\
\text { da polpa }(\%)\end{array}$ & $\begin{array}{c}\text { Frutos } \\
\text { podres }(\%)\end{array}$ \\
\hline Análise inicial & - & $17,4 \pm 2,4$ & $0,106 \pm 0,030$ & $10,4 \pm 0,6$ & - & - \\
\hline Controle & $15,09 \mathrm{a}^{*}$ & $13,8 \mathrm{bc}$ & $0,098 \mathrm{a}$ & 8,9 a & $27,8 \mathrm{a}$ & $8,3 \mathrm{ab}$ \\
\hline PEBD $40 \mu \mathrm{m}$ & $0,29 \mathrm{~b}$ & $16,9 \mathrm{a}$ & $0,078 \mathrm{~b}$ & $8,3 \mathrm{a}$ & $0,0 \mathrm{~b}$ & $35,0 \mathrm{a}$ \\
\hline PEBD $60 \mu \mathrm{m}$ & $0,39 \mathrm{~b}$ & $11,9 \mathrm{c}$ & $0,086 \mathrm{~b}$ & $9,0 \mathrm{a}$ & $8,3 \mathrm{~b}$ & $16,7 \mathrm{ab}$ \\
\hline PEBD $90 \mu \mathrm{m}$ & $0,26 \mathrm{~b}$ & $13,6 \mathrm{bc}$ & $0,080 \mathrm{~b}$ & $8,4 \mathrm{a}$ & $0,0 \mathrm{~b}$ & $15,0 \mathrm{ab}$ \\
\hline PEMD $40 \mu \mathrm{m}$ & $0,28 \mathrm{~b}$ & $14,7 \mathrm{ab}$ & $0,085 \mathrm{~b}$ & $8,7 \mathrm{a}$ & $0,0 \mathrm{~b}$ & $25,0 \mathrm{ab}$ \\
\hline PEMD $60 \mu \mathrm{m}$ & $0,28 \mathrm{~b}$ & $12,4 \mathrm{bc}$ & $0,081 \mathrm{~b}$ & $8,5 \mathrm{a}$ & $6,9 \mathrm{~b}$ & $0,0 \mathrm{~b}$ \\
\hline Média geral & 2,80 & 13,9 & 0,085 & 8,6 & 7,2 & 16,7 \\
\hline CV (\%) & 37,83 & 11,55 & 7,53 & 5,57 & 58,37 & 76,83 \\
\hline
\end{tabular}

${ }^{1}$ PEBD - polietileno de baixa densidade; PEMD - polietileno de média densidade.

*Médias seguidas pela mesma letra, na vertical, não diferem entre si pelo teste Duncan em $5 \%$ de probabilidade de erro.

após o período de armazenamento, não diferindo estatisticamente dos frutos armazenados em PEMD de $40 \mu \mathrm{m}$ (Tabela 2). Esse resultado pode estar associado às menores concentrações de etileno no interior da embalagem de PEBD de $40 \mu \mathrm{m}$ (Figura 1C). Segundo HADFIELD et al. (1998), as pectinas representam a principal classe de polissacarídeos das paredes celulares, sendo solubilizadas e despolimerizadas durante o amadurecimento, o que resulta na redução da firmeza da polpa dos frutos. A degradação enzimática das paredes celulares em melões é um evento controlado pelo etileno (SILVA et al., 2004). LIMA et al. (2000), trabalhando com maçãs, observaram que, com o uso de atmosfera controlada com absorção de etileno, o processo de solubilização praticamente não ocorreu durante o período de armazenamento, mantendo uma maior firmeza da polpa.

Maior acidez titulável foi observada nos frutos não embalados, não ocorrendo efeito significativo dos filmes de polietileno avaliados (Tabela 2). Esse efeito pode estar relacionado à desidratação dos frutos, que deve ter concentrado os ácidos nos frutos não embalados. O teor de ácidos orgânicos apresenta pouca contribuição para o aroma e o sabor do melão, em função de sua baixa concentração (MENEZES, 1996).

Os teores de SST decresceram durante o período de armazenamento, porém não houve diferença estatística entre os tratamentos (Tabela 2). O teor de açúcares, que compõem $90 \%$ dos SST, é dependente do tempo em que o fruto fica preso à planta (WELLES \& BUITELAAR, 1988). No entanto, após os frutos serem colhidos, os teores de SST começam a decrescer
(SILVA et al., 2004), provavelmente devido ao aumento no consumo dos açúcares pelo metabolismo respiratório.

A ocorrência de degenerescência da polpa foi reduzida significativamente com o uso de filmes de polietileno, possivelmente em razão de menor perda de massa (Tabela 2). Já a incidência de podridões foi significativamente menor nos frutos armazenados em PEMD de $60 \mu \mathrm{m}$ (Tabela 2). Segundo SEYMOUR \& McGLASSON (1993), melões reticulados são bastante suscetíveis ao ataque fúngico durante o transporte e a comercialização. Menor população microbiana foi observada por BAI et al. (2003) em melões minimamente processados e armazenados em atmosfera modificada passiva e ativa, provavelmente em virtude de as baixas concentrações de $\mathrm{O}_{2}$ e as altas concentrações de $\mathrm{CO}_{2}$ terem retardado o crescimento microbiano. No presente trabalho, observou-se a condensação de vapor de água nas embalagens de PEBD e PEMD, demonstrando uma baixa permeabilidade desses filmes à perda de água, $\mathrm{o}$ que deve ter possibilitado o desenvolvimento de lesões fúngicas, mesmo sob altas concentrações de $\mathrm{CO}_{2}$.

\section{CONCLUSÕES}

Os filmes avaliados mantiveram de modo semelhante a qualidade físico-química de melões híbrido Torreon produzidos hidroponicamente no sistema NFT. $\mathrm{O}$ uso de filmes plásticos reduz drasticamente a perda de massa de melões e a degenerescência da polpa, quando comparados aos não embalados.

Em relação aos filmes avaliados neste trabalho, observou-se que a espessura e a densidade 

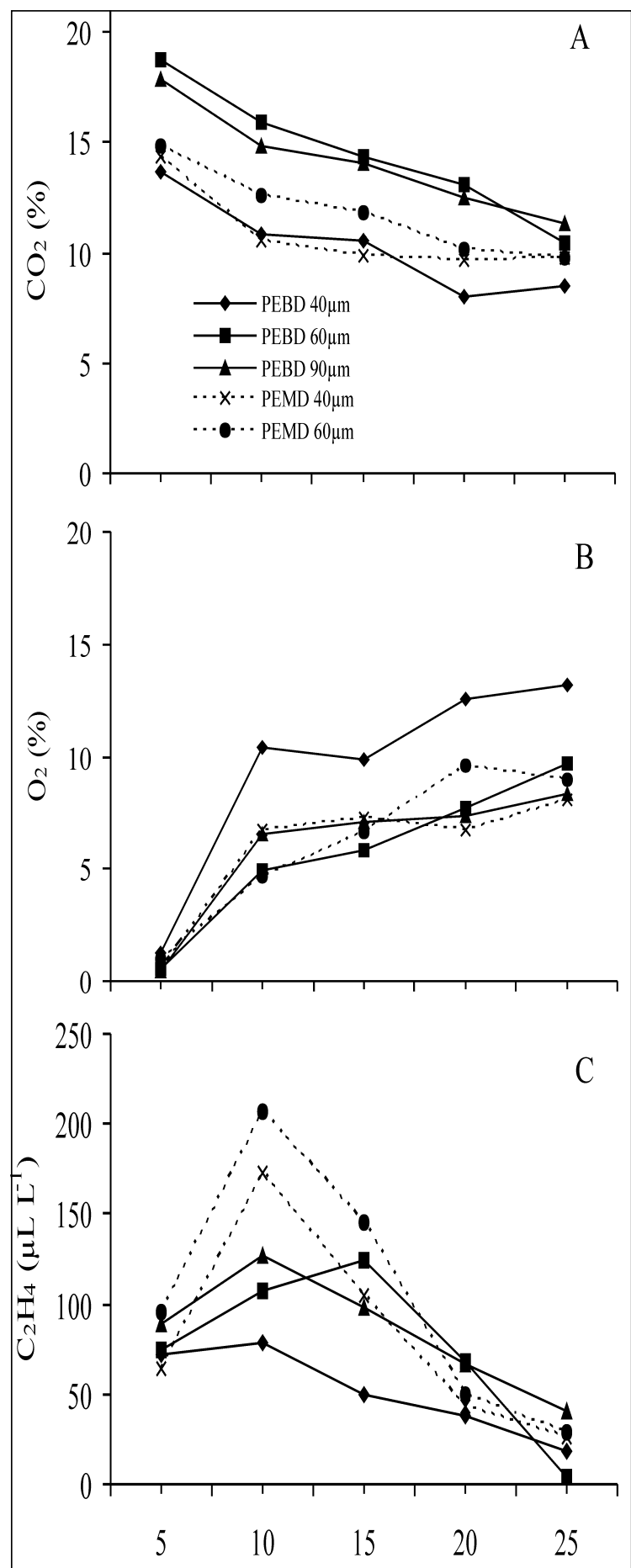

Dias a $3,8 \pm 0,2^{\circ} \mathrm{C}$

Figura 1 - Concentrações de $\mathrm{CO}_{2}(\mathrm{~A})$, de $\mathrm{O}_{2}$ (B) e de $\mathrm{C}_{2} \mathrm{H}_{4}$ em embalagens de polietileno de diferentes espessuras e densidades contendo cinco melões var. cantalupensis híbrido Torreon, com $970 \pm 130 \mathrm{~g}$ cada e armazenados a $3,8 \pm 0,2^{\circ} \mathrm{C}$. Santa Maria, RS. 2004 do filme apresentam maior efeito sobre a permeabilidade ao $\mathrm{O}_{2}$, sendo a permeabilidade ao $\mathrm{CO}_{2}$ aparentemente mais afetada pela espessura do filme plástico, que pela sua densidade. Durante 25 dias de armazenamento de melões híbrido Torreon a $3,8 \pm 0,2^{\circ} \mathrm{C}$ e $87 \pm 3 \%$ de UR do ar, os filmes de PEBD de 60 e $90 \mu \mathrm{m}$ e o PEMD de $60 \mu \mathrm{m}$ permitiram maiores concentrações de $\mathrm{CO}_{2}$ nas embalagens, sendo que o filme de PEBD de $40 \mu \mathrm{m}$ resultou em maior concentração de $\mathrm{O}_{2}$ e menor de etileno. Sugere-se a condução de novos trabalhos visando a avaliar o efeito da absorção do etileno na conservação de melões.

\section{AGRADECIMENTOS}

Ao Conselho Nacional de Desenvolvimento Científico e Tecnológico (CNPq), pela concessão de bolsa aos pesquisadores Eisermann e Giehl. À Fundação de Amparo à Pesquisa do Estado de São Paulo (FAPESP), pela concessão de bolsa ao pesquisador Fagan. À Coordenação de Aperfeiçoamento de Pessoal de Nível Superior (CAPES), pela concessão de bolsa ao pesquisador Steffens.

\section{REFERÊNCIAS}

BAI, J. et al. Characteristics of fresh-cut honeydew (Cucumis $\mathrm{x}$ melo L.) available to processors in winter and summer and its quality maintenance by modified atmosphere packaging. Postharvest Biology and Technology, Amsterdam, v.28, p.349-359, 2003.

BEAUDRY, R.M. Effect of $\mathrm{O}_{2}$ and $\mathrm{CO}_{2}$ partial pressure on selected phenomena affecting fruit and vegetable quality. Postharvest Biology and Technology, Amsterdam, v.15, p.293-303, 1999.

BRACKMANN, A. et al. Efeito de filmes de PVC esticável e polietileno no acúmulo de $\mathrm{CO}_{2}$ e na manutenção da qualidade pós-colheita de morangos cv. Tangi. Revista Brasileira de Agrociência, Pelotas, v.5, n.2, p.89-92, 1999.

FAGAN, E.B. Regime de irrigação e densidade de frutos na produção do melão hidropônico. 2005. 60f. Dissertação (Mestrado em Agronomia) - Curso de Pós-graduação em Agronomia, Universidade Federal de Santa Maria.

HADFIELD, K.A. et al. Polygalacturonase gene expression in ripe melon fruit supports a role for polygalacturonase in ripening-associated pectin disassembly. Plant Physiology, Lancaster, v.117, n.2, p.363-373, 1998.

HARDENBURG, R.E. et al. Commercial storage of fruits, vegetables and florist and nursery stocks. Beltsville: USDA-ARS, 1986. 136p.

KADER, A.A.; KE, D. Controlled atmospheres. In: PAULL, R.E.; ARMSTRONG, J.W. (Ed). Insect pest and fresh horticultural products: treatment and responses. Wallingford: CAB International, 1994. p.223-236. 
LIMA, L.C. et al. Perda de firmeza da polpa de maçãs (Malus domestica, Borkh.) Royal Gala armazenadas sob refrigeração e atmosfera controlada. Revista Brasileira de Fruticultura, Jaboticabal, v.22, n. especial, p.26-31, 2000.

MAYBERRY, K.S.; HARTZ, T.K. Extension of muskmelon storage life through the use of hot water treatment and polyethylene wraps. HortScience, Alexandria, v.27, n.4, p.24236, 1992.

MENEZES, J.B. Qualidade pós-colheita de melão tipo Gália durante a maturação e armazenamento. 1996. 157f. Tese (Doutorado em Ciência dos Alimentos) - Curso de Pós-graduação em Ciência dos Alimentos, Universidade Federal de Lavras.

PORTELA, S.I.; CANTWELL, M.I. Quality changes of minimally processed honeydew melons stored in air or controlled atmosphere. Postharvest Biology and Technology, Amsterdam, v.14, p.351-357, 1998.
SARANTÓPOULOS, C.I.G.L. et al. Embalagens plásticas flexíveis: principais polímeros e avaliação de propriedades. Campinas: CETEA/ITAL, 2002. 267p.

SEYMOUR, G.B.; McGLASSON, W.B. Melons. In: SEYMOUR, G.B. et al. (Ed). Biochemistry of fruit ripening. London: Chapman \& Hall, 1993. p.273-290.

SILVA, J.A. et al. Characterization of ripening behavior in transgenic melons expressing an antisense 1 aminocyclopropane-1-carboxilate (ACC) oxidase gene from apple. Postharvest Biology and Technology, Amsterdam, v.32, p.263-268, 2004.

WELLES, G.W.H.; BUITELAAR, K. Factor affecting soluble solids content of muskmelon (Cucumis melo L.). Netherlands Journal of Agricultural Science, Amsterdam, v.36, p.239246, 1988 . 